\title{
PASSIVE LINEAR DISCRETE-TIME SYSTEMS: CHARACTERIZATION THROUGH STRUCTURE
}

\author{
IZCHAK LEWKOWICZ
}

\begin{abstract}
We here show that the family of finite-dimensional, discrete-time, passive, linear time-invariant systems can be characterized through the structure of a matrixconvex set, which is maximal in the sense of being closed under products of its elements Moreover, this observation unifies three setups: (i) difference inclusions, (ii) matrix-valued rational functions, (iii) realization arrays associated with rational functions.

It turns out that in the continuous-time case the corresponding structure is of a maximal matrix-convex cone closed under inversion.
\end{abstract}

AMS Classification: 15A60 26C15 47L07 47A56 47N70 93B15

Key words: matrix-convex sets, discrete-time bounded real rational functions, passive linear systems, state-space realization, Kalman-Yakubovich-Popov Lemma

\section{Contents}

1. Introduction

2. Sets of Matrices with Common Stein Factor

3. Maximal multiplicative matrix-convex sets of matrices

4. Multiplicative Matrix-convex sets of Rational Functions

5. Sets of Matrix-convex Realization Arrays

References

\section{INTRODUCTION}

In the study of dynamical systems, passivity is a fundamental property. Thus, it has been extensively addressed in various frameworks. A fundamental contribution was made by J.C. Willems, see e.g. [30], 31] and [32. Here we confine the discussion to discrete-time systems. More precisely, we focus on the finite-dimensional, linear time-invariant case. These passive systems are modelled by Discrete-time Bounded real rational functions, denoted by $\mathcal{D B}$, namely (here, for simplicity, in a scalar framework) rational functions which map $\{z \in \mathbb{C}:|z|>1\}$, the exterior of the closed unit disk, to $\{z \in \mathbb{C}: 1 \geq|z|\}$, the closed unit disk. (In the sequel, the discussion is of matrix-valued rational functions).

In reading relevant literature, some extra care is quite helpful:

Here we follow the engineering motivation where $F(z)$ can be interpreted as the $Z$ transform, i.e. $F(z)=C\left(z I_{n}-A\right)^{-1} B+D$, of the shift-invariant difference state-equation $x(k+1)=A x(k)+B u(k), y(k)=C x(k)+D u(k)$, where in some sense, the input $u$ dominates the output $y$, i.e. $\|u\| \geq\|y\|$ for all input $u$. For more general formulation see e.g. [15]. 
In contrast, apparently motivated by symmetry, mathematical-analysis circles prefer to study Schur functions which analytically map the open unit disk $\{z \in \mathbb{C}: 1>|z|\}$ to its closure, $\{z \in \mathbb{C}: 1 \geq|z|\}$, see e.g. [11] and [27]. This family was for example addressed in [1] and [9].

On the top of this difficulty, there is a confusion in names of various associated families of functions. See e.g. [3, Section 2], 20] and [21]. We shall not further pursue this point.

There have been characterizations of finite-dimensional discrete-time passive systems. For a modest sample of the literature on the subject, see e.g. [24], 26], [29] and [33, Section 4]. For an account of the infinite-dimensional case, see e.g. [6], 7], 8], [9] and [28].

Here we adopt a more abstract point of view and focus on the following question:

How can one characterize the family of finite-dimensional, discrete-time, passive, linear time-invariant systems through the structure of the whole set?

The answer is that this family forms a matrix-convex set which is maximal with respect to being closed under products among its elements. Moreover, this observation unifies three setups:

(i) Difference inclusions,

(ii) Discrete-time Bounded real rational functions,

(iii) Families of realization arrays of $\mathcal{D B}$, Discrete-time Bounded real rational functions.

This is a follow-up of the study in [19] of the continuous-time case. Combining the message of both works can be summarized as follows,

\begin{tabular}{|c|c|}
\hline Passive linear time-invariant systems and matrix-convexity \\
\hline discrete-time & continuous-time \\
\hline $\begin{array}{c}\text { a maximal set closed under } \\
\text { products of its elements }\end{array}$ & $\begin{array}{c}\text { a cone closed under inversion and } \\
\text { maximal non-singular/analytic }\end{array}$ \\
\hline
\end{tabular}

This work is organized as follows. In Section 2 we lay the foundation to the sequel and recall in sets of matrices all satisfying a Stein inclusion with the same factor. Then, in Section 3 we restrict the discussion to matrix-convex sets of matrices which are closed under products among its elements and maximal in this sense, see Proposition 3.4. As a sample motivation we recall in the problem of stability of difference inclusions.

Subsequently, in Section 4 we exploit Proposition 3.4 to characterize, in Proposition 4.2 , Discrete-time Bounded, $\mathcal{D B}$, real rational functions. Then the same structure is used to describe, in Corollary 5.3, families of realization arrays, of $\mathcal{D B}$ functions.

The conclusion that passive discrete-time systems (even of various dimensions) are interrelated, is illustrated in Examples 4.1 and 5.4, for rational functions and for realization arrays, respectively.

\section{Sets of Matrices with Common Stein Factor}

We start with notations. Let $\overline{\mathbf{H}}_{n}\left(\mathbf{H}_{n}\right)$ be the set of $n \times n$ Hermitian (non-singular) matrices and by $\left(\overline{\mathbf{P}}_{n}\right) \mathbf{P}_{n}$ denote the subsets of $n \times n$ positive (semi)-definite matrices. 
Now, for a prescribed $H \in \mathbf{H}_{n}$, consider the set of all $n \times n$ matrices $A$ sharing the same Stein factor,

$$
\begin{aligned}
& \mathbf{S}_{H}=\left\{A \in \mathbb{C}^{n \times n}: H-A^{*} H A \in \mathbf{P}_{n}\right\} \\
& \overline{\mathbf{S}}_{H}=\left\{A \in \mathbb{C}^{n \times n}: H-A^{*} H A \in \overline{\mathbf{P}}_{n}\right\}
\end{aligned} \quad H \in \mathbf{H}_{n} .
$$

The set $\overline{\mathbf{S}}_{H}$ is the closure of the open set $\mathbf{S}_{H}$ in the sense that $\overline{\mathbf{P}}_{n}$ is the closure in $\overline{\mathbf{H}}_{n}$ of the open set $\mathbf{P}_{n}$.

One can refine the above definition by adding a parameter $\alpha>0$ to obtain, $\overline{\mathbf{S}}_{H}$ ),

$$
\begin{aligned}
& \frac{1}{\alpha} \mathbf{S}_{H}=\left\{A \in \mathbb{C}^{n \times n}: H-\frac{1}{\alpha^{2}} A^{*} H A \in \mathbf{P}_{n}\right\} \\
& \frac{1}{\alpha} \overline{\mathbf{S}}_{H}=\left\{A \in \mathbb{C}^{n \times n}: H-\frac{1}{\alpha^{2}} A^{*} H A \in \overline{\mathbf{P}}_{n}\right\}
\end{aligned} \quad H \in \mathbf{H}_{n} .
$$

We now examine the structure of this set.

Theorem 2.1. For $H \in \mathbf{H}_{n}$ the set $\mathbf{S}_{H}\left(\frac{1}{\alpha} \overline{\mathbf{S}}_{H}\right)$ is open (closed) convex closed under multiplication by $c \in \mathbb{C}, 1 \geq|c|$ and closed under products of its elements. Furthermore, whenever $A \in \frac{1}{\alpha} \mathbf{S}_{H}$ and $B \in \frac{1}{\beta} \mathbf{S}_{H}$, for some $\alpha, \beta>0$, then the product of these matrices satisfies $A B \in \frac{1}{\alpha \beta} \mathbf{S}_{H}$.

Proof : Although classical, for completeness we show this for $\frac{1}{\alpha} \mathbf{S}_{H}$. Convexity, closure under multiplication by $c \in \mathbb{C}, 1 \geq|c|$ and the fact that this set is open, are all trivial.

Assume that for some $H \in \mathbf{H}_{n}$ one has that $A \in \frac{1}{\alpha} \mathbf{S}_{H}$ and $B \in \frac{1}{\beta} \mathbf{S}_{H}$, for some $\alpha, \beta>0$ namely,

$$
\begin{array}{ll}
H-\frac{1}{\alpha^{2}} A^{*} H A=Q_{a} & \text { for some } \\
H-\frac{1}{\beta^{2}} B^{*} H B=\mathbf{P}_{n}
\end{array}
$$

Multiplying the first equation by $\frac{1}{\beta} B^{*}$ and $\frac{1}{\beta} B$ from the left and from the right respectively, and adding the result to the second equation yields,

$$
H-\frac{1}{\alpha^{2} \beta^{2}}(A B)^{*} H A B=\frac{1}{\beta^{2}} B^{*} Q_{a} B+Q_{b},
$$

and as the right hand side is positive definite.

Establishing this claim for the closed set $\frac{1}{\alpha} \overline{\mathbf{S}}_{H}$ is analogous is thus omitted.

In the sequel, we focus our attention on the case where in Eq. (2.2) one has that $H \in \mathbf{P}_{n}$.

Corollary 2.2. Consider the description in Theorem [2.1 of the set $\frac{1}{\alpha} \mathbf{S}_{H}$ in Eq. (2.2). Whenever $H \in \mathbf{P}_{n}$ this is in addition a family of matrices whose spectral radius is bounded by $\alpha$.

Indeed, when $H \in \mathbf{P}_{n}$, one can multiply the Stein matrix inclusion in Eq. (2.2) by $H^{-\frac{1}{2}}$ from both sides to obtain,

$$
\begin{array}{ll}
\frac{1}{\alpha} \mathbf{S}_{H}=\left\{A \in \mathbb{C}^{n \times n}: \alpha>\left\|H^{\frac{1}{2}} A H^{-\frac{1}{2}}\right\|_{2}\right\} & H \in \mathbf{P}_{n} \\
\frac{1}{\alpha} \overline{\mathbf{S}}_{H}=\left\{A \in \mathbb{C}^{n \times n}: \alpha \geq\left\|H^{\frac{1}{2}} A H^{-\frac{1}{2}}\right\|_{2}\right\} & \alpha>0 .
\end{array}
$$

Thus, in particular, the spectral radius of $A$ is bounded by $\alpha$. 
We conclude this section by pointing out that a complete characterization of the set $\mathbf{S}_{H}$ in Eq. (2.1), for an arbitrary $H \in \mathbf{H}_{n}$, appeared in [5, Theorem 3.5]. This remarkable result is quite involved. Now, on the expense of restricting the case to $H=I_{n}$, in Proposition 3.4 below, we obtain, through matrix-convexity, a much simpler characterization. Subsequently, this advantage is exploited to describe Discrete-time Bounded real rational functions.

\section{Maximal multiplicative matriX-CONVEX Sets of Matrices}

We next resort to the notion of a matrix-convex set, see e.g. [12] and more recently, [13], [14], [17], [25].

Definition 3.1. A family $\mathbf{A}$, of square matrices (of various dimensions) is said to be matrixconvex, if for all natural $k, n$,

$$
\sum_{j=1}^{k} v_{j}^{*} v_{j}=I_{n} \quad \forall v_{j} \in \mathbb{C}^{\eta_{j} \times n}
$$

one has that having $A_{1}, \ldots, A_{k}$ (of dimensions $\eta_{1} \times \eta_{1}$ through $\eta_{k} \times \eta_{k}$ ) within $\mathbf{A}$, implies that also the $n \times n$ matrix

$$
\sum_{j=1}^{k} v_{j}^{*} A_{j} v_{j}
$$

belongs to $\mathbf{A}$.

In the sequel, Skew-Hermitian matrices are denoted by, $i \overline{\mathbf{H}}$. It is common to take $\overline{\mathbf{H}}$ and $i \overline{\mathbf{H}}$ as the matricial extension of $\mathbb{R}$ and $i \mathbb{R}$, respectively.

Remark 3.2. In [19] it was shown that there are not-too-many, non-trivial matrix-convex sets, among them: $\overline{\mathbf{H}}, \quad i \overline{\mathbf{H}}, \quad \overline{\mathbf{P}}, \quad \mathbf{P}$.

Note that matrix-convexity is rather stringent. Specifically, by definition, matrix-convexity implies both classical convexity and having the set invariant under all unitary similarities. The following Example 3.3 illustrates the fact that the converse falls short from being true.

Example 3.3. a. Following Remark 3.2, for arbitrary $\alpha>0$, the subset of all matrices in $\overline{\mathbf{H}}$ (or within $i \overline{\mathbf{H}}$ or within $\overline{\mathbf{P}}$, or within $\mathbf{P}$ ) whose spectral radius is less or equal to $\alpha>0$, is matrix convex.

b. The family of matrices whose Frobenius (a.k.a. Euclidean or Hilbert-Schmidt) norm, see e.g. [18, p. 291] is bounded say by some $\alpha>0$, is both convex and unitarily invariant, but it is not matrix-convex.

Consider the set of matrices $\left\{A: 5 \geq\|A\|_{\text {Frobenius }}\right\}$. Now from $A=\left(\begin{array}{ll}4 & 0 \\ 0 & 3\end{array}\right)$ which belongs to this set $\left(\|A\|_{\text {Frobenius }}=5\right)$, construct the matrix

$$
\hat{A}=\underbrace{\left(\begin{array}{llll}
1 & 0 & 0 & 0 \\
0 & 0 & 1 & 0
\end{array}\right)}_{\Upsilon^{*}}\left(\begin{array}{cc}
A & 0 \\
0 & A
\end{array}\right) \underbrace{\left(\begin{array}{ll}
1 & 0 \\
0 & 0 \\
0 & 1 \\
0 & 0
\end{array}\right)}_{\Upsilon}=4 I_{2} .
$$

Now, since $\|\hat{A}\|_{\text {Frobenius }}=4 \sqrt{2} \approx 5.7$, this set is not matrix-convex. 
We find it convenient to use, for a prescribed $\alpha>0$, this notation,

$$
\frac{1}{\alpha} \mathbf{S}_{I}=\bigcup_{n=1}^{\infty} \frac{1}{\alpha} \mathbf{S}_{I_{n}} \quad \text { and } \quad{ }_{\frac{1}{\alpha}} \overline{\mathbf{S}}_{I}=\bigcup_{n=1}^{\infty} \frac{1}{\alpha} \overline{\mathbf{S}}_{I_{n}} .
$$

We next present the key player in this work.

Proposition 3.4. A closed (open), matrix-convex family of matrices whose spectral radius is less or equal to some $\alpha>0$, containing (on its boundary) all matrices of the form $\left(\begin{array}{cc}\alpha e^{i \theta} & 0 \\ 0 & 0\end{array}\right)$ with $\theta \in[0,2 \pi)$, is the set $\frac{1}{\alpha} \overline{\mathbf{S}}_{I}\left(\frac{1}{\alpha} \mathbf{S}_{I}\right)$.

Furthermore, the converse is true as well.

If in addition this is a maximal family of matrices which (whenever dimensions are suitable) is closed under products among its elements, then it is equivalent to having

$$
\alpha=1 \text {. }
$$

Proof: First as matrix-convex sets are in particular closed under unitary similarity, from the family in the claim, we actually obtain all $2 \times 2$ rank one normal matrices with spectral radius $\alpha$. Now, taking matrix-convex combinations, yields all (not necessarily normal) $2 \times 2$ matrices with spectral radius $\alpha$. Taking convex combination with zero, results in all $2 \times 2$ matrices with spectral radius of at most $\alpha$.

Let now $U \in \mathbb{C}^{n \times 2}$, where $n \geq 3$, be an arbitrary isometry, i.e. $U^{*} U=I_{2}$. Multiplying a matrix $A \in \mathbb{C}^{2 \times 2}$ from the above set, by $U A U^{*}$ yields $n \times n$ matrices of degree of at most two, with spectral radius of at most $\alpha$. Taking further matrix-convex combination recovers all possible degrees up to (including) $n$.

To show that this is indeed $\overline{\mathbf{S}}_{I}$ (or $\mathbf{S}_{I}$ ) recall that from Eq. (2.3)

$$
{ }_{\alpha}^{1} \mathbf{S}_{I_{n}}=\left\{A \in \mathbb{C}^{n \times n}: \alpha>\|A\|_{2}\right\} \quad \text { and } \quad \frac{1}{\alpha} \overline{\mathbf{S}}_{I_{n}}=\left\{A \in \mathbb{C}^{n \times n}: \alpha \geq\|A\|_{2}\right\} .
$$

Next, recall that for every induced norm, a set of the form $\left\{A \in \mathbb{C}^{n \times n}: \alpha>\|A\|\right\}$, is convex and the spectral radius of all matrices in it, is bounded by $\alpha$, see e.g. [18, Section $5.6]$.

To guarantee matrix-convexity, the norm must be in addition unitarily-invariant, which implies \|\|$_{2}$.

Next, we show that the closed set $\frac{1}{\alpha} \overline{\mathbf{S}}_{I_{n}}$ (the case of the open set $\frac{1}{\alpha} \mathbf{S}_{I_{n}}$ is similar and thus omitted) is matrix-convex. For a natural parameter $k$ let $\Upsilon \in \mathbb{C}^{k n \times n}$ be an isometry, i.e. $\Upsilon^{*} \Upsilon=I_{n}$, then

$$
\begin{aligned}
& \left\|\Upsilon^{*}\left(\begin{array}{ccc}
A_{1} & & \\
& \ddots & \\
& & \\
& A_{k}
\end{array}\right) \Upsilon\right\|_{2} \leq\left\|\Upsilon^{*}\right\|_{2}\left\|\left(\begin{array}{ccc}
A_{1} & & \\
& \ddots & \\
& & A_{k}
\end{array}\right)\right\|_{2}\|\Upsilon\|_{2} \quad \text { sub - multiplicative norm } \\
& =\left\|\left(\begin{array}{ccc}
A_{1} & & \\
& \ddots & \\
& & A_{k}
\end{array}\right)\right\|_{2} \quad \Upsilon \text { is an isometry } \\
& =\max \left(\left\|A_{1}\right\|_{2}, \ldots,\left\|A_{k}\right\|_{2}\right) \quad \text { induced norm } \\
& \leq \quad \alpha \quad \text { assumption, }
\end{aligned}
$$


so this part of the claim is established.

For maximality of the spectral norm under product of elements, let $B \notin \overline{\mathbf{S}}_{I_{n}}$ be arbitrary. One can always find, within $\mathbf{S}_{I_{n}}$, a matrix $A$ so that the spectral radius of the product $A B$, is larger than one (and thus the spectral radius of $(A B)^{l}$ is diverging with $l$ natural). Indeed, let the Singular Value Decomposition, see e.g. [18, Theorem 7.35], of a matrix $B$ be

$$
B=\sum_{j=1}^{n} \sigma_{j} u_{j} v_{j}^{*} \quad \begin{array}{ccc}
(1+\epsilon)=\sigma_{1} \geq \sigma_{2} \geq \cdots \geq \sigma_{n} \geq 0, \quad \epsilon>0 \\
u_{j} \in \mathbb{C}^{n} & u_{j}^{*} u_{k}=\delta_{j, k} & n \geq j \geq k \geq 1 \\
v_{j} \in \mathbb{C}^{n} & v_{j}^{*} v_{k}=\delta_{j, k} & n \geq j \geq k \geq 1
\end{array}
$$

where $\delta_{j, k}$ is the Kronecker delta. To avoid triviality, assume that the spectral radius of $B$ is less than one (Schur stable). This implies that $\frac{1}{1+\epsilon}>\left|u_{1} v_{1}^{*}\right|$ (when $\sigma_{2}=0$, this is in fact sufficient).

Take now $A=\frac{1}{1+2 \epsilon} B^{*}$. By construction $\|A\|_{2}=\frac{1+\epsilon}{1+2 \epsilon}$, so indeed $A \in \mathbf{S}_{I_{n}}$. Next,

$$
A B=\frac{1}{1+2 \epsilon} B^{*} B=\frac{1}{1+2 \epsilon} \sum_{j=1}^{n} \sigma_{j}^{2} v_{j} v_{j}^{*}=\frac{1}{1+2 \epsilon}\left((1+\epsilon)^{2} v_{1} v_{1}^{*}+\sum_{j=2}^{n} \sigma_{j}^{2} v_{j} v_{j}^{*}\right) .
$$

Thus, in fact $A B \in \overline{\mathbf{P}}_{n}$ and $\|A B\|_{2}=\frac{(1+\epsilon)^{2}}{1+2 \epsilon}=1+\frac{\epsilon^{2}}{1+2 \epsilon}$, which is also the spectral radius of $A B$, so this part of the construction is complete.

The converse direction is to show that the set $\frac{1}{\alpha} \mathbf{S}_{I_{n}}$ is of this structure. This is easy and thus omitted.

Finally, to obtain a set which is closed under products of its elements, one needs to take $1 \geq \alpha$, and maximality requires $1=\alpha$. Thus the proof is complete.

As an application consider the following, see e.g. [23].

\section{Stability of difference inclusions}

Recall that the solution $x(j)$ of an autonomous difference equation $x(j+1)=A x(j)$ converges to zero for all $x(0)$, if and only if the spectral radius of $A$ is less than one. Recall also that the set of matrices whose spectral radius is less than one (colloquially, "Schur stable") is not closed under multiplication, e.g. both matrices $A=\left(\begin{array}{ll}0 & 2 \\ 0 & 0\end{array}\right)$ and $B=A^{*}$, have a zero spectral radius. However, the spectral radius of the product $A B$, is four.

Let $\mathbf{M}$ be a given set of real $n \times n$ matrices. A difference inclusion

$$
x(j+1) \in \mathbf{M} x(j) \quad \begin{gathered}
x(j) \in \mathbb{R}^{n} \\
j=0,1,2, \ldots
\end{gathered}
$$

can be interpreted as having

$$
x(j+1)=A(j) x(j) \quad j=0,1,2, \ldots A(j) \text { is }\left\{\begin{array}{l}
\text { arbitrary } \\
\text { within } \mathbf{M} .
\end{array}\right.
$$

From Proposition 3.4 it follows that:

Corollary 3.5. There exists $\alpha \in(0,1-\epsilon]$, with $1>>\epsilon>0$, so that the difference inclusion in Eq. (3.3) satisfies Eq. (3.4),

$$
\|x(0)\|_{2} \alpha^{j} \geq\|x(j)\|_{2} \quad \forall j=0,1,2, \ldots
$$


if and only if for the same $\alpha$,

$$
\mathbf{M} \subset \frac{1}{\alpha} \overline{\mathbf{S}}_{I_{n}}
$$

For completeness we recall that if for some $\alpha \in(0,1-\epsilon]$, with $1>>\epsilon>0$ the condition is relaxed to $\mathbf{M} \subset\{A: \alpha>\|A\|\}$, for some induced matrix norm, see e.g. [18, Section 5.6], then Eq. (3.4) holds when \|\|$_{2}$ is substituted by the above induced norm 11 .

In the next section we use the set $\mathbf{S}_{I_{n}}$ to describe a family of rational functions.

\section{Multiplicative Matrix-Convex sets of Rational Functions}

In this section we address Discrete-time Bounded real $m \times m$-valued rational functions $F(z)$, denoted by $\mathcal{D} \mathcal{B}$, satisfying

$$
\left(I_{m}-(F(z))^{*} F(z)\right) \in \overline{\mathbf{P}}_{m} \quad \forall z \in \mathbb{C} \text { s.t. }|z|>1 .
$$

See e.g. [21], [24], [26] and [29].

Note that Eq. (4.1) can be equivalently written as,

$$
1 \geq\|F(z)\|_{2} \quad \forall z \in \mathbb{C} \text { s.t. }|z|>1 .
$$

As already mentioned, $\mathcal{D B}$ functions can be interpreted as the $Z$-transform of the shiftinvariant difference state equation,

$$
x(k+1)=A x(k)+B u(k) \quad y(k)=C x(k)+D u(k),
$$

i.e. $F(z)=C\left(z I_{n}-A\right)^{-1} B+D$, where in the sense of Eq. (4.2), the input $u$ dominates the output $y$, i.e. $\|u\| \geq\|y\|$ for all input $u$.

Eqs. (4.1) and (4.2) in particular imply that whenever $F_{a}$ and $F_{b}$ are two $m \times m$-valued rational functions, with this property, then so is their product $F_{a} F_{b}$.

Applying the notation of Proposition 3.4, the set of $\mathcal{D B}$ functions $F(z)$ in Eq. (4.2), can be equivalently written as $m \times m$-valued rational functions $F(z)$ so that 2 ,

$$
F(z) \in\left\{\begin{array}{c}
\mathbb{R}^{m \times m} \quad z \in \mathbb{R} \\
\overline{\mathbf{S}}_{I_{m}} \quad z \in \mathbb{C} \text { s.t. }|z|>1 .
\end{array}\right.
$$

We now illustrate matrix-convexity operations among matrix-valued rational functions, to be used in the sequel.

Example 4.1. Let $F_{1}(z), F_{2}(z)$ and $F_{3}(z)$ be rational functions of dimensions $1 \times 1,2 \times 2$ and $3 \times 3$, respectively. From these functions, by taking matrix-convex operations, one can construct functions $G_{1}(z), G_{2}(z)$ and $G_{3}(z)$, of dimensions $1 \times 1,2 \times 2$ and $3 \times 3$, respectively. (To ease reading, the isometric matrices are partitioned conform ably with

\footnotetext{
${ }^{1}$ In principle this can further relaxed in two ways: (i) to having possibly another norm and (ii) $\beta \geq 1$ so that $\beta\|x(0)\| \alpha^{j} \geq\|x(j)\| \forall j=0,1,2, \ldots$

${ }^{2}$ Strictly speaking the first line in Eq. (4.3) should be read as saying that: "Whenever $z \in \mathbb{R}$ is not a pole of $F\left((z)\right.$, then $F(z) \in \mathbb{R}^{m \times m}$."
} 
$F_{1}, F_{2}$ and $\left.F_{3}\right)$ :

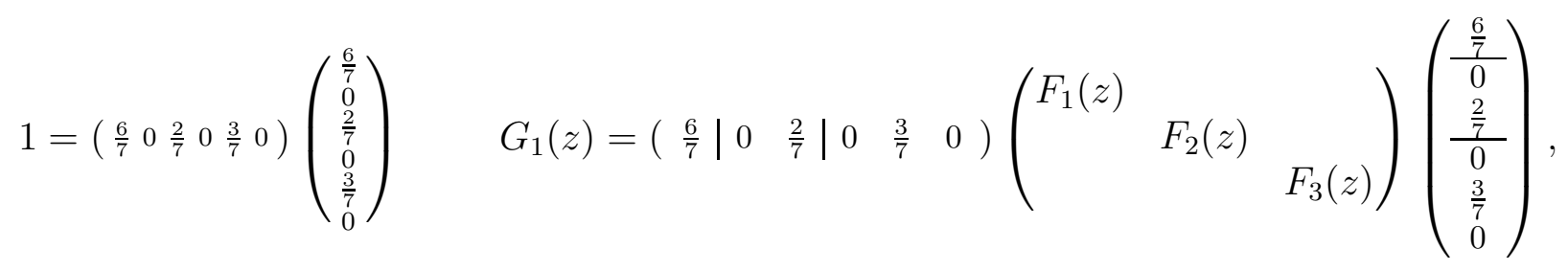

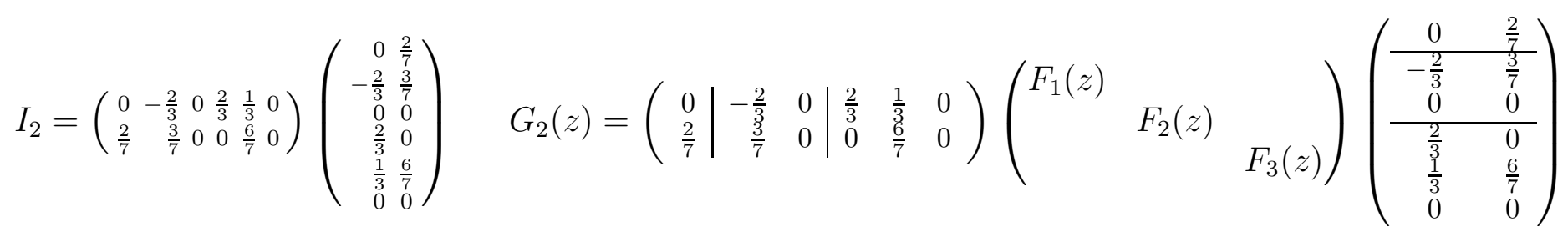

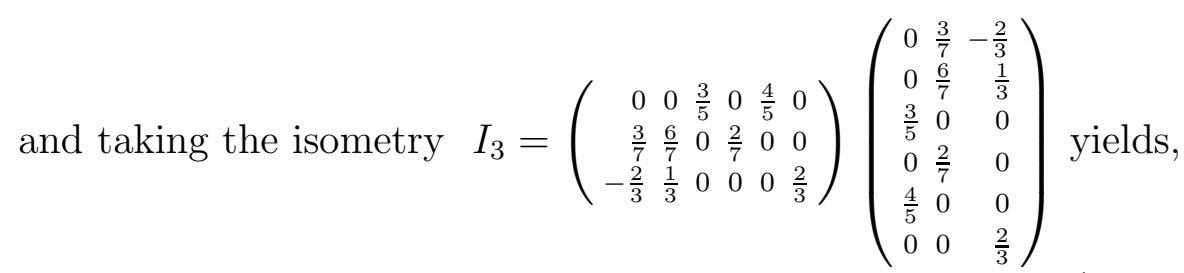

$G_{3}(z)=\left(\begin{array}{r|cc|ccc}0 & 0 & \frac{3}{5} & 0 & \frac{4}{5} & 0 \\ \frac{3}{7} & \frac{6}{7} & 0 & \frac{2}{7} & 0 & 0 \\ -\frac{2}{3} & \frac{1}{3} & 0 & 0 & 0 & \frac{2}{3}\end{array}\right)\left(\begin{array}{lll}F_{1}(z) & & \\ & F_{2}(z) & \\ & & F_{3}(z)\end{array}\right)\left(\begin{array}{ccc}0 & \frac{3}{7} & -\frac{2}{3} \\ \hline 0 & \frac{6}{7} & \frac{1}{3} \\ \frac{3}{5} & 0 & 0 \\ \hline 0 & \frac{2}{7} & 0 \\ \frac{4}{5} & 0 & 0 \\ 0 & 0 & \frac{2}{3}\end{array}\right)$.

Using Eq. (4.3) along with Proposition [3.4, we have the following characterization of rational $\mathcal{D B}$ functions.

Proposition 4.2. Let $\mathcal{F}$ be a family of square matrix-valued (of various dimensions) real rational functions $F(z)$. For all $z$ outside the closed unit disk, each $F(z)$ is analytic.

If as a family, $\mathcal{F}$ is matrix-convex $\mathbf{3}^{3}$ and a maximal set closed under products of its elements (whenever dimensions are suitable), this is the set $\mathcal{D B}$ of Discrete-time Bounded real rational functions.

The converse is true as well.

As we already mentioned, matrix-convexity is a strong property. This is next illustrated in the context of $\mathcal{D B}$ functions.

Example 4.3. If $F_{1}(z), F_{2}(z)$ and $F_{3}(z)$ in Example 4.1 are $\mathcal{D B}$ functions then so are $G_{1}(z), G_{2}(z)$ and $G_{3}(z)$.

Remark 4.4. To simplify the exposition, we consider square matrix-valued rational functions. However, some of the results are carried over to the rectangular case where,

$$
\overline{\mathbf{S}}_{I_{m, p}}=\left\{A \in \mathbb{C}^{p \times m}: I_{m}-A^{*} A \in \overline{\mathbf{P}}_{m}\right\}
$$

\footnotetext{
${ }^{3}$ In the sense described in Example 4.1.
} 
and then a $p \times m$-valued rational $\mathcal{D B}$ function $F(z)$ can be described as 4 ,

$$
F(z) \in\left\{\begin{array}{l}
\mathbb{R}^{p \times m} \quad z \in \mathbb{R} \\
\overline{\mathbf{S}}_{I_{m, p}} \quad z \in \mathbb{C} \text { s.t. }|z|>1 .
\end{array}\right.
$$

For more details see e.g. [24], and for a subclass of $\mathcal{D B}$ functions see [2].

To simplify the exposition, we avoid this generalization.

In the next section we study the structure of families of realization arrays associated with $\mathcal{D B}$ functions.

\section{Sets of Matrix-Convex Realization Arrays}

Recall that whenever $F(z)$ is an $m \times m$-valued rational function with no pole at infinity, one can associate with it a corresponding $(n+m) \times(n+m)$ state-space realization array, $R_{F}$ i.e.

$$
F(z)=C\left(z I_{n}-A\right)^{-1} B+D \quad R_{F}=\left(\begin{array}{c|c}
A & B \\
\hline C & D
\end{array}\right) .
$$

The realization $R_{F}$ in Eq. (5.1) is called minimal, if $n$ is the McMillan degree of $F(z)$.

In this section we address families of realization arrays associated with rational functions. To this end, we adopt the an idea apparently from [31, Section 5] to treat the above $(n+m) \times(n+m) R_{F}$ as having two faces: 5 : $i$ ) of an array and (ii) of a matrix. (For recent applications of matrix manipulations of $R_{F}$ see [3], [19], [20] and [22]).

Before that, a word of caution: For example, $R_{1}=\left(\begin{array}{r|r}A & B \\ \hline C & D\end{array}\right)$ and $R_{2}=\left(\begin{array}{r|r}A & -B \\ \hline-C & D\end{array}\right)$ are two realization of the same rational function. Furthermore, $R_{1}$ is minimal (balanced) if and only if $R_{2}$ is minimal (balanced). However, $R_{3}=\frac{1}{2}\left(R_{1}+R_{2}\right)=\left(\begin{array}{c|c}A & 0 \\ \hline 0 & D\end{array}\right)$ is a realization of a zero degree rational function $F(s) \equiv D$.

To further study families of realizations of $\mathcal{D B}$ functions, we need to introduce a relaxed version of matrix-convexity.

Definition 5.1. For all $k$, let $v_{j} \in \mathbb{C}^{(n+m) \times(n+m)}, j=1, \ldots, k$ be block-diagonal so that

$$
\sum_{j=1}^{k} \underbrace{\left(\begin{array}{cc}
v_{j, n} & 0 \\
0 & v_{j, m}
\end{array}\right)^{*}}_{v_{j}^{*}} \underbrace{\left(\begin{array}{cc}
v_{j, n} & 0 \\
0 & v_{j, m}
\end{array}\right)}_{v_{j}}=\left(\begin{array}{cc}
I_{n} & 0 \\
0 & I_{m}
\end{array}\right) .
$$

A set $\mathbf{R}$, of $(n+m) \times(n+m)$ matrices, is said to be $n, m$-matrix-convex if having $R_{F_{1}}, \ldots, R_{F_{k}}$ in $\mathbf{R}$, implies that also,

$$
\sum_{j=1}^{k} \underbrace{\left(\begin{array}{cc}
v_{j, n} & 0 \\
0 & v_{j, m}
\end{array}\right)^{*}}_{v_{j}^{*}} \underbrace{\left(\begin{array}{cc}
A_{j} & B_{j} \\
C_{j} & D_{j}
\end{array}\right)}_{R_{F_{j}}} \underbrace{\left(\begin{array}{cc}
v_{j, n} & 0 \\
0 & v_{j, m}
\end{array}\right)}_{v_{j}},
$$

\footnotetext{
${ }^{4}$ Strictly speaking the first line below should be read as saying thet: "Whenever $z \in \mathbb{R}$ is not a pole of $F\left((z)\right.$, then $F(z) \in \mathbb{R}^{p \times m}$."

${ }^{5}$ Like Janus in the Roman mythology
} 
belongs to $\mathbf{R}$, for all natural $k$ and all block-diagonal $v_{j} \in \mathbb{C}^{(n+m) \times(n+m)}$.

In [19] it was pointed out that the notion of $n$, m-matrix-convexity is intermediate between (the more strict) matrix-convexity, and (weaker) classical convexity.

For a natural parameter $k$, let $F_{1}(z), \ldots, F_{k}(z)$ be a family of $m \times m$-valued rational functions whose $(n+m) \times(n+m)$ realizations are 6 ,

$$
R_{F_{j}}=\left(\begin{array}{c|c}
\hat{A}_{j} & \hat{B}_{j} \\
\hline \hat{C}_{j} & \hat{D}_{j}
\end{array}\right) \quad j=1, \ldots, k .
$$

Using block-diagonal structured isometries from Eq. (5.2) along with the realizations $R_{F_{j}}$ in Eq. (5.3), let $R_{F}$ be of the form,

$$
R_{F}=\sum_{j=1}^{k} \underbrace{\left(\begin{array}{cc}
v_{j, n} & 0 \\
0 & v_{j, m}
\end{array}\right)^{*}}_{v_{j}^{*}} \underbrace{\left(\begin{array}{cc}
\hat{A}_{j} & \hat{B}_{j} \\
\hat{C}_{j} & \hat{D}_{j}
\end{array}\right)}_{R_{F_{j}}} \underbrace{\left(\begin{array}{cc}
v_{j, n} & 0 \\
0 & v_{j, m}
\end{array}\right)}_{v_{j}} .
$$

Let now $F(z)$ be an $m \times m$-valued rational function whose realization $R_{F}$ is given by Eq. (5.4). We now address the following problem: Under what conditions does having the functions $F_{1}(z), \ldots, F_{k}(z)$, in Eq. (5.3) , Discrete-time-Bounded real, imply that the resulting $F(z)$ in Eq. (5.4) is $\mathcal{D B}$ as well?

If such a property holds, this suggests that out of a small number of "extreme points" of balanced realizations of $\mathcal{D B}$ rational functions, one can construct a whole "matrixconvex-hull" of realizations of functions, within the same family. This may enable one to perform a simultaneous balanced truncation model order reduction of a whole family of $\mathcal{D B}$ functions, in the spirit of [10, Section 5].

As already indicated, even when the "extreme points" realizations are balanced, the resulting "intermediate" realization may be not minimal.

Recall that the classical version of the Kalman-Yakubovich-Popov Lemma for Discretetime Bounded real rational functions, see e.g. [24], [26], [29] and [33, Section 4], says the following.

Lemma 5.2. Let $F(z)$ be an $m \times m$-valued rational function and let $R_{F}$ be a corresponding realization see (5.1)

(I) If there exists a matrix $P \in \mathbf{P}_{n}$ so that

$$
\left(\begin{array}{cc}
P & 0 \\
0 & I_{m}
\end{array}\right)-\underbrace{\left(\begin{array}{cc}
A & B \\
C & D
\end{array}\right)^{*}}_{R_{F}^{*}}\left(\begin{array}{cc}
P & 0 \\
0 & I_{m}
\end{array}\right) \underbrace{\left(\begin{array}{cc}
A & B \\
C & D
\end{array}\right)}_{R_{F}} \in \overline{\mathbf{P}}_{n+m},
$$

then $F(z)$ is a $\mathcal{D} \mathcal{B}$ function.

If $F(z)$ is in $\mathcal{D B}$ function and its realization in Eq. (5.1), is minimal, i.e. $n$ is the McMillan degree, then Eq. (5.5) is satisfied.

(II) Up to change of coordinates, one can substitute in Eq. (5.5) $P=I_{n}$ so that,

$$
\left(\begin{array}{cc}
I_{n} & 0 \\
0 & I_{m}
\end{array}\right)-\underbrace{\left(\begin{array}{cc}
A & B \\
C & D
\end{array}\right)^{*}}_{R_{F}^{*}}\left(\begin{array}{cc}
I_{n} & 0 \\
0 & I_{m}
\end{array}\right) \underbrace{\left(\begin{array}{cc}
A & B \\
C & D
\end{array}\right)}_{R_{F}} \in \overline{\mathbf{P}}_{n+m} .
$$

\footnotetext{
${ }^{6}$ As poles are all within the unit disk, these realizations do exist.
} 
In particular, this is the case when the realization is balanced.

Combining Proposition 3.4 along with part (II) of Lemma 5.2 we can now answer the question posed in the beginning of this section.

Corollary 5.3. For a natural parameter $k$, let $F_{1}(z), \ldots, F_{k}(z)$ be a family of $m \times m$-valued rational functions. Assume that they all admit $(n+m) \times(n+m)$ realizations as in Eqs. (5.3), (5.6), i.e.

$$
\left(\begin{array}{cc}
I_{n} & 0 \\
0 & I_{m}
\end{array}\right)-\underbrace{\left(\begin{array}{cc}
A_{j} & B_{j} \\
C_{j} & D_{j}
\end{array}\right)^{*}}_{R_{F_{j}}^{*}}\left(\begin{array}{cc}
I_{n} & 0 \\
0 & I_{m}
\end{array}\right) \underbrace{\left(\begin{array}{cc}
A_{j} & B_{j} \\
C_{j} & D_{j}
\end{array}\right)}_{R_{F_{j}}} \in \overline{\mathbf{P}}_{n+m} \quad j=1, \ldots, k .
$$

Then, an arbitrary realization $R_{F}$ defined by Eq. (5.4), satisfies Eq. (5.6) and thus the associated $F(z)$, is a rational $\mathcal{D B}$ function.

A different formulation (and a different proof) of a similar result appeared in [20, Proposition 5.3].

The fact that realization arrays of $\mathcal{D B}$ rational functions are inter-related, is next illustrated.

Example 5.4. We here show how by matrix manipulations of realization arrays of Discrete time Bounded rational functions, one can "generate" a whole family of such functions.

1. For parameters $\theta \in(0,1)$ and $a>1$ consider the following scalar $\mathcal{D B}$ rational function of degree one,

$$
f_{1}(z)=\theta \cdot \frac{a+z}{a z+1} \quad R_{f_{1}}=\frac{1}{a}\left(\begin{array}{c|c}
-1 & \sqrt{\theta\left(a^{2}-1\right)} \\
\hline \sqrt{\theta\left(a^{2}-1\right)} & \theta
\end{array} .\right.
$$

Treating $R_{f_{1}}$ as a $2 \times 2$ matrix, let us define $R_{f_{2}}:=\left(\begin{array}{cc}1 & 0 \\ 0 & -1\end{array}\right) R_{f_{1}}\left(\begin{array}{cc}-1 & 0 \\ 0 & 1\end{array}\right)$ so that,

$$
f_{2}(z)=\theta \cdot \frac{a-z}{a z-1} \quad R_{f_{2}}=\frac{1}{a}\left(\begin{array}{c|c}
1 & \sqrt{\theta\left(a^{2}-1\right)} \\
\hline \sqrt{\theta\left(a^{2}-1\right)} & -\theta
\end{array} .\right.
$$

is another Discrete-time Bounded scalar rational functions of degree one.

For example, taking now $R_{f_{3}}:=\frac{1}{2}\left(R_{f_{1}}+R_{f_{2}}\right)$ yields yet another $\mathcal{D B}$ rational functions of degree one,

$$
f_{3}(z)=\frac{\theta}{a^{2}} \cdot \frac{a^{2}-1}{z} \quad R_{f_{3}}=\sqrt{\theta\left(1-\frac{1}{a^{2}}\right)}\left(\begin{array}{c|c}
0 & 1 \\
\hline 1 & 0
\end{array}\right) .
$$

Note that each of the realization $R_{f_{1}}, R_{f_{2}}$ and $R_{f_{3}}$ is balanced and satisfies Eq. (5.7).

The following product, $f_{4}(z):=f_{1}(z) f_{2}(z)$, is a $\mathcal{D B}$ rational function of degree two,

$$
f_{4}(z)=\theta^{2} \cdot \frac{a^{2}-z^{2}}{a^{2} z^{2}-1} \quad R_{f_{4}}=\frac{1}{a}\left(\begin{array}{cc|c}
-1 & \frac{\theta}{a}\left(1-a^{2}\right) & \frac{\theta}{a} \sqrt{\theta\left(a^{2}-1\right)} \\
0 & 1 & \sqrt{\theta\left(a^{2}-1\right)} \\
\hline-\sqrt{\theta\left(a^{2}-1\right)} & \frac{\theta}{a} \sqrt{\theta\left(a^{2}-1\right)} & -\frac{\theta^{2}}{a}
\end{array}\right) .
$$

Now, $\hat{R}_{f_{4}}:=\left(\begin{array}{rrr}0 & -1 & 0 \\ 1 & 0 & 0 \\ 0 & 0 & 1\end{array}\right) R_{f_{4}}\left(\begin{array}{rrr}0 & 1 & 0 \\ -1 & 0 & 0 \\ 0 & 0 & 1\end{array}\right)$ is another minimal realization of the same $f_{4}(z)$.

Let now define $R_{f_{5}}:=\frac{1}{2}\left(R_{f_{4}}+\hat{R}_{f_{4}}\right)$. 
For simplicity of presentation take $\theta=\frac{1}{2}$ and $a=3$ then, out of $f_{4}(z)$, whose poles are $\pm \frac{1}{3}$, one obtains the following $\mathcal{D B}$ function, of degree two,

$$
f_{5}(z)=\frac{1}{36} \cdot \frac{\left(\frac{4}{3}\right)^{2}-z^{2}}{z^{2}+\left(\frac{2}{9}\right)^{2}} \quad R_{f_{5}}=\frac{1}{36}\left(\begin{array}{rr|r}
0 & -8 & -10 \\
8 & 0 & 14 \\
\hline 14 & 10 & 1
\end{array}\right)
$$

whose poles are $\pm \frac{2}{9} i$.

2. In a way similar to the above, we next illustrate how by taking matrix products and matrix-convex operations, one can "generate" virtually all realization arrays of a scalar $\mathcal{D B}$ rational of McMillan degree of at most 1.

Indeed, substituting in Eq. (5.6) $n=1$ and $m=1$, reveals that one can take $R_{f_{j}}(j$ natural) to be a $2 \times 2$ orthogonal matrix (with det $=1$ ) of the form

$$
f_{j}(z)=\frac{z \cos \left(\theta_{j}\right)-1}{z-\cos \left(\theta_{j}\right)} \quad R_{f_{j}}:=\left(\begin{array}{rr}
\cos \left(\theta_{j}\right) & -\sin \left(\theta_{j}\right) \\
\sin \left(\theta_{j}\right) & \cos \left(\theta_{j}\right)
\end{array}\right) \quad \begin{aligned}
& j=1,2, \ldots \\
& \theta_{j} \in[0,2 \pi) .
\end{aligned}
$$

Recall also that for all $\theta_{1}$ and $\theta_{2}$,

$$
R_{f_{1}} R_{f_{2}}=\left(\begin{array}{rr}
\cos \left(\theta_{1}+\theta_{2}\right) & -\sin \left(\theta_{1}+\theta_{2}\right) \\
\sin \left(\theta_{1}+\theta_{2}\right) & \cos \left(\theta_{1}+\theta_{2}\right)
\end{array}\right)
$$

which can be viewed as both: another orthogonal matrix of the form of Eq. (5.9) and a balanced realization another $\mathcal{D B}$ function satisfying Eq. (5.6) with zero right hand side, colloquially para-unitary, see e.g. [2].

For simplicity, take now an arbitrary $\theta_{1}=\theta_{2}$ provided that $\frac{\theta_{1}}{\pi}$ is irrational (e.g. $\theta_{1}$ rational). This means that by taking infinite powers of the form $\left(R_{f_{1}}\right)^{k}, k=1,2, \ldots$ one obtains a dense subset of all $2 \times 2$ orthogonal matrices of the from of Eq. (5.9).

Next take the matrix $\tilde{R}=\left(\begin{array}{rr}-1 & 0 \\ 0 & 1\end{array}\right)$. $\tilde{R}$ can also be viewed as a (non-minimal) realization of the zero degree rational function $f(z) \equiv 1$. As matrices, we have,

$$
\underbrace{\left(\begin{array}{rr}
-1 & 0 \\
0 & 1
\end{array}\right)}_{\tilde{R}} \underbrace{\left(\begin{array}{rr}
\cos \left(\theta_{j}\right) & -\sin \left(\theta_{j}\right) \\
\sin \left(\theta_{j}\right) & \cos \left(\theta_{j}\right)
\end{array}\right)}_{R_{f_{j}}}=\underbrace{\left(\begin{array}{rr}
-\cos \left(\theta_{j}\right) & \sin \left(\theta_{j}\right) \\
\sin \left(\theta_{j}\right) & \cos \left(\theta_{j}\right)
\end{array}\right)}_{R_{\hat{f}_{j}}} .
$$

For each $j=1,2, \ldots$ the right hand side, $R_{\hat{f}_{j}}$ is a symmetric orthogonal matrix (with det $=-1$ ) and a realization of,

$$
\hat{f}_{j}(z)=\frac{z \cos \left(\theta_{j}\right)+1}{z+\cos \left(\theta_{j}\right)} \quad j=1,2, \ldots
$$

Finally, note that $f_{j}(z)$ and $\hat{f}_{j}(z)$ are weak contractions. Taking matrix-convex combinations of $R_{f_{j}}$ and of $R_{\hat{f}_{j}}$, yields strict contractions. Thus, the sought construction of all arrays is complete.

3. Recall that in principle the idea of item 2 can be carried over to higher dimensions, by taking products of planar rotations (a.k.a. Givens rotations, see e.g. [18, Example 2.2.3]) of the form,

$$
\left(\begin{array}{ccc}
\cos \left(\theta_{1}\right) & -\sin \left(\theta_{1}\right) & 0 \\
\sin \left(\theta_{1}\right) & \cos \left(\theta_{1}\right) & 0 \\
0 & 0 & 1
\end{array}\right)\left(\begin{array}{ccc}
\cos \left(\theta_{2}\right) & 0 & -\sin \left(\theta_{2}\right) \\
0 & 1 & 0 \\
\sin \left(\theta_{2}\right) & 0 & \cos \left(\theta_{2}\right)
\end{array}\right)\left(\begin{array}{ccc}
1 & 0 & 0 \\
0 & \cos \left(\theta_{3}\right) & -\sin \left(\theta_{3}\right) \\
0 & \sin \left(\theta_{3}\right) & \cos \left(\theta_{3}\right)
\end{array}\right) \quad \theta_{1}, \theta_{2}, \theta_{3} \in[0,2 \pi) .
$$

4. We conclude by pointing out that one can be more adventurous in manipulating realization arrays. Take for instance $R_{f_{4}}$ from Eq. (5.8). By construction, as a matrix it 
satisfies

$$
\left(I_{3}-R_{f_{4}}^{*} R_{f_{4}}\right) \in \overline{\mathbf{P}}_{3} .
$$

Now, another partitioning of $R_{f_{4}}$ corresponds to a $2 \times 2$-valued $\mathcal{D B}$ function which is of McMillan degree one,

$F_{6}(z)=\frac{1}{a}\left(\begin{array}{cc}1 & \sqrt{\theta\left(a^{2}-1\right)} \\ \theta \sqrt{\theta\left(a^{2}-1\right)} \cdot \frac{z+a}{a z+1} & -\frac{\theta^{2}}{a} \cdot \frac{z+a}{a z+1}\end{array}\right) \quad R_{F_{6}}=\frac{1}{a}\left(\begin{array}{ccc}-1 & \frac{\theta}{a}\left(1-a^{2}\right) & \frac{\theta}{a} \sqrt{\theta\left(a^{2}-1\right)} \\ \hline-\sqrt{\theta\left(a^{2}-1\right)} & \frac{\theta}{a} \sqrt{\theta\left(a^{2}-1\right)} & -\frac{\theta^{2}}{a}\end{array}\right)$.

Finally, clearly $\left(\begin{array}{ll}0 & 1\end{array}\right) F_{6}(z)\left(\begin{array}{l}0 \\ 1\end{array}\right)$ is a scalar $\mathcal{D} \mathcal{B}$ function of McMillan degree one. In fact it equals to $-\frac{\theta}{a} \cdot f_{1}(z)=-\frac{\theta^{2}}{a^{2}} \cdot \frac{z+a}{z+\frac{1}{a}}$.

\section{ACKNOWLEDGEMENT}

The constructive and exceptionally thorough review, is highly appreciated.

\section{REFERENCES}

[1] D. Alpay, A. Dijksma, J. Rovnyak and H. de Snoo, "Schur Functions and Their Canonical Realizations", pp. 41-82 in Schur Functions Operator Colligation and Reproducing Kernel Pontryagin Spaces, Vol. 96 of Operator Theory: Advances and Applications, Birkhäser, Basel, 1997.

$[2]$ D. Alpay, P.E.T. Jorgensen and I. Lewkowicz, "Characterizations of Families of Rectangular, Finite Impulse Response, Para-Unitary Systems", Journal of Applied Mathematics and Computing Vol. 54, pp. 395-423, 2017.

[3] D. Alpay and I. Lewkowicz, "The Positive Real Lemma and Construction of all Realizations of Generalized Positive Rational Functions", Systems and Control Letters, Vol. 60, pp. 985-993, 2011.

[4] D. Alpay and I. Lewkowicz, "Quantitatively Hyper-Positive Real rational functions", see arXiv:1912-08245.

[5] T. Ando, "Sets of Matrices with Common Stein Solutions and $H$-contractions", Linear Algebra and its Applications, Vol. 383, pp. 49-64, 2004.

[6] Y.M. Arlinskii, S. Hassi, H.V. de Snoo, "Parametrization of Contractive Block Operator Matrices and Passive Discrete-Time Systems", Complex Analysis and Operator Theory, Vol. 1, pp. 211-233, 2007.

[7] D.Z. Arov and O.J. Staffans, "State/Signal Linear Time-Invariant Systems Theory, Part I: DiscreteTime Systems", The State Space Method Generalization and Applications, Vol. 161 of Operator Theory: Advances and Applications, pp. 115-177, Birkhäser, Basel, 2005.

[8] J.A. Ball, G.J. Groenewald and S. ter Horst, "Standard versus Strict Bounded Real Lemma with Infinite-Dimensional State Space II: The Storage Function Approach", pp. 1-50 in The Diversity and Beauty of Applied Operator Theory, Vol. 268 of Operator Theory Advances and Applications, Birkhäuser Verlag, Basel; Birkhäuser Verlag, Basel, 2018.

[9] J.A. Ball and O.J. Staffans, "Conservative State-Space Realizations of Dissipative System Behaviors", Integral Equations and Operator Theory, Vol. 54, pp. 151-213, 2005.

[10] N. Cohen and I. Lewkowicz, "Convex Invertible Cones of State Space Systems", Mathematics of Control Signals and Systems, Vol. 10, pp. 265-285, 1997.

[11] L. de Branges and J. Rovnyak, Square Summable Power Series, Holt, Reinhart and Wilson, 1966.

[12] E.G. Effros and S. Winkler, "Matrix Convexity: Operator Analogues of the Bipolar and Han-Banach Theorems", Journal of Functional Analysis, Vol. 144, pp. 117-152, 1997.

[13] E. Evert, "Matrix Convex Sets Without Absolute Extreme Points", Linear Algebra and its Applications, Vol. 537, pp. 287-301, 2018.

[14] E. Evert, J.W. Helton, I. Klep and S. McCullough, "Extreme Points of Matrix Convex Sets, Free Spectrahedra and Dilation Theory", Journal of Geometric Analysis, Vol. 28, pp. 1373-1408, 2018. 
[15] I. Goethas, T. Van Gestel, J. Suykens, P. Van Dooren and B. De Moor, "Identification of Positive Real Models in Subspace Identification by Using Regularization", IEEE Transaction on Automatic Control, Vol. 48, pp.1843-1847, 2003.

[16] J.W. Helton, S. McCullough and V. Vinnikov, "Non-commutative Convexity Arises from Linear Matrix Inequalities", Journal of Functional Analysis, Vol. 240, pp. 105-191, 2006.

[17] J.W. Helton, I. Klep and S. McCullough, "The Matricial Relaxation of a Linear Matrix Inequality" Math. Program., Vol. 138, pp. 401-445, 2013.

[18] R. A. Horn and C. R. Johnson, Matrix Analysis, Cambridge University Press, 1985.

[19] I. Lewkowicz, "Passive Linear Continuous-time Systems: Characterization through Structure", to appear in Systems and Control Letters, arXiv:1912.08249.

[20] I. Lewkowicz, "A Unified Framework for Continuous/Discrete Positive/Bounded Real State-Space Systems", arXiv:2008.04635.

[21] M. Liu and J. Xiong, "Bilinear Transformation for Discrete-Time Positive Real and Negative Imaginary Systems", IEEE Transaction on Automatic Control, Vol. 63, pp. 4264-4269, 2018.

[22] V. Mehrmann and P. Van Dooren, "Optimal Robustness of Passive Discrete-Time Systems", IMA Journal of Mathematics and Information, pp. 1-22, 2020.

[23] A.P. Molchanov and Ye.S. Pyatnitskiy, "Criteria of Asymptotic Stability of Differential and Difference Inclusions Encountered in Control Theory", Systems and Control Letters, Vol. 13, pp. 59-64, 1989.

[24] F. Najson, "On the Kalma-Yakobovich-Popov Lemma for Discrete-Time Positive Linear Systems: A Novel Simple Proof and Some Related Results", International Journal of Control, Vol. 86, pp. 1813-1823, 2013.

[25] B. Passer, O. Shalit and B. Solel, "Minimal and Maximal Matrix Convex Sets", Journal of Functional Analysis, Vol. 274, pp. 3197-3253, 2018.

[26] K. Premaratne and E.I. Jury, "Discrete-Time Positive Real Lemma Revisited: The Discrete-Time Counterpart of the Kalman-Yakubotich Lemma", IEEE Transaction on Circuits and Systems I: Fundamental Theory and Applications, Vol. 41, pp. 740-743, 1994.

[27] "Schur Functions in Complex Function Theory, Encyclopedia of Mathematics", URL : http ://www.encyclopediaofmath.org/index.php/Schur_functions_in_complex_function_theory

[28] O. Staffans, "Passive Linear Discrete Time-Invariant Systems", International Congress of Mathematicians, European Mathematical Society, Zurich 2006, Vol. 3, pp. 1367-1388.

[29] V.V. Vaidyanathan, "The Discrete-Time Bounded Real Lemma in Digital Filtering", IEEE Transaction on Circuits and Systems, Vol. 42, pp. 918-924, 1985.

[30] J.C. Willems, "Dissipative Dynamical Systems Part I: General Theory", Archive for Rational Mechanics and Analysis, Vol. 45, pp. 321-351, 1972.

[31] J.C. Willems, "Dissipative Dynamical Systems Part II: Linear Systems with Quadratic Supply Rate", Archive for Rational Mechanics and Analysis, Vol. 45, pp. 352-393, 1972.

[32] J.C. Willems, "Realization of Systems with Internal Passivity and Symmetry Constraints", Journal of the Franklin Institute, Vol. 301, pp. 605-621, 1976.

[33] C. Xiao and D.J. Hill, "Generalization and New Proof of the Discrete-Time Positive Real Lemma and Bounded Real Lemma", IEEE Transaction on Circuits and Systems I: Fundamental Theory and Applications, Vol. 46, pp. 740-743, 1999.

School of Electrical and Computer Engineering Ben-Gurion University of the Negev, P.O.B. 653, BEER-Sheva, 84105, IsRAEL

Email address: izchak@bgu.ac.il 